\title{
Comparison of plasma PUFA concentration between supplement users and non-supplement users in Irish adults
}

\author{
K. F. Li $^{1}$, B. A. McNulty ${ }^{1}$, A. M. Tierney ${ }^{1}$, F. J. Bloomfield ${ }^{2}$, D. J. Duff ${ }^{2}$, M. J. Gibney ${ }^{1}$ \\ and A. P. Nugent ${ }^{1}$ \\ ${ }^{1}$ UCD Institute of Food and Health, University College Dublin, Belfield, Dublin 4, Ireland and ${ }^{2}$ Chemical Analysis \\ Laboratories, Dublin, Ireland
}

The $n-3$ polyunsaturated fatty acid (PUFA) is a family of essential fats that humans have limited ability to synthesize de novo ${ }^{(1)}$. Nutrition supplements from fish and plant oils are often used to enhance dietary intakes of these fats. However, the relationship between supplement use and plasma fatty acids concentration in Irish adults is poorly defined. The objective of the study was to explore the impact of supplement use on plasma PUFA concentration in a representative sample of Irish adults using data from the National Adult Nutrition Survey (NANS) ${ }^{(2)}$.

The NANS estimated habitual food and beverage intake in a representative sample of 1,500 Irish adults. A 4-day semi-weighed food diary was used to record food and beverage intake data and an assessment of PUFA intake was performed using manufacture's information and published food composition databases. Consenting participants provided a fasting blood sample after the diary recording period had ended ${ }^{(2)}$. Plasma total fatty acids were extracted and transesterified using Bligh \& Dyer ${ }^{(3)}$ and Ohta methods ${ }^{(4)}$, respectively. For the purpose of this analysis consumers of supplements containing PUFA and non-supplement users, who provide a blood sample $(n=1076)$ were divided into two separate groups and differences in plasma PUFA were explored by independent $t$-tests using SPSS version 20.0 (IBM Inc. Chicago, USA).

Plasma concentration of eicosadienoic acid, eicosatrienoic acid and docosatetraenoic acid were significantly lower in supplement users than in non-supplement users. In contrast, plasma concentration of total PUFA, eicosapentaenoic acid (EPA), docosapentaenoic acid (DPA) and docosahexaenoic acid (DHA) were significantly higher in supplement users than those in non-supplement users. This reflected dietary intake data which indicated that EPA, DHA and $\alpha$-linolenic aicd (ALA) intake were significantly $(P<0.001)$ higher among supplement users $(3.472 \pm 10.512 \mathrm{~g} / \mathrm{d}, \quad 3.411 \pm 10.280 \mathrm{~g} / \mathrm{d}$ and $1.808 \pm 1.692 \mathrm{~g} / \mathrm{d}$, respectively) than non-supplement users $(0.091 \pm 0.147 \mathrm{~g} / \mathrm{d}, 0.140 \pm 0.232 \mathrm{~g} / \mathrm{d}$ and $1.303 \pm 0.695 \mathrm{~g} / \mathrm{d}$, respectively). Plasma ALA concentration did not differ between groups perhaps due to elongation to EPA, DPA and DHA ${ }^{(5)}$. In conclusion, supplementation with $n-3$ PUFA can increase plasma concentrations of long-chain PUFA in healthy Irish adults.

\begin{tabular}{|c|c|c|c|c|c|}
\hline \multirow[b]{2}{*}{ Plasma fatty acids (mg/mL) } & \multicolumn{2}{|c|}{ Supplement users $(n=131)$} & \multicolumn{2}{|c|}{ Non-supplement users $(n=945)$} & \multirow[b]{2}{*}{$\mathrm{p} 1$} \\
\hline & Mean & SD & Mean & SD & \\
\hline Total PUFA & 1.395 & 0.284 & 1.364 & 0.283 & ns \\
\hline$n-6$ PUFA & 1.162 & 0.252 & 1.173 & 0.244 & ns \\
\hline Linoleic acid (C18:2n6c) & 0.825 & 0.184 & 0.834 & 0.186 & $\mathrm{~ns}$ \\
\hline$\gamma$-Linolenic $(\mathrm{C} 18: 3 n 6)$ & 0.018 & 0.008 & 0.019 & 0.008 & ns \\
\hline Eicosadienoic acid (C20:2) & 0.011 & 0.005 & 0.013 & 0.007 & $*$ \\
\hline Eicosatrienoic acid (C20:3n6) & 0.062 & 0.020 & 0.067 & 0.022 & $*$ \\
\hline Arachidonic acid (C20:4n6) & 0.250 & 0.103 & 0.246 & 0.071 & ns \\
\hline Docosatetraenoic acid $(\mathrm{C} 22: 4 n 6)$ & 0.007 & 0.003 & 0.008 & 0.004 & $* *$ \\
\hline$n-3$ PUFA & 0.222 & 0.083 & 0.178 & 0.067 & $* * *$ \\
\hline$\alpha$-Linolenic aicd $(\mathrm{C} 18: 3 n 3)$ & 0.028 & 0.012 & 0.028 & 0.013 & ns \\
\hline Eicosatetraenoic acid (C20:4n3) & 0.006 & 0.005 & 0.006 & 0.004 & $\mathrm{~ns}$ \\
\hline Eicosapentaenoic acid (C20:5n3) & 0.062 & 0.041 & 0.043 & 0.027 & $* * *$ \\
\hline Docosapentaenoic acid (C22:5n3) & 0.029 & 0.010 & 0.027 & 0.009 & $* *$ \\
\hline Docosahexaenoic acid (C22:6n3) & 0.096 & 0.037 & 0.075 & 0.032 & $* * *$ \\
\hline
\end{tabular}

${ }^{1}$ Independent $t$-test of means between supplement and non-supplement users $* P<0.05, * * P<0.01$, $* * * P<0.001$, ns: not significant.

The project was funded by the Department of Agriculture, Fisheries and Food and the Health Research Board under the Food for Health Research Initiative 2007-2012.

1. Arterburn LM, Hall EB, Oken H. (2006) Am J Clin Nutr 83, 1467S-76S

2. National Adult Nutrition Survey Summary Report (2011) Available at: http://www.iuna.net/wp-content/uploads/2010/12/National-Adult-Nutrition-Survey-Summary-Report-March-2011.pdf (Accessed at 04/03/2013).

3. Bligh EG, Dyer WJ. (1959) Can J Bioc Phys 37, 911-7.

4. Morrison WR, Smith LM. (1964) J Lipid Res 5, 600-8.

5. Burdge GC, Calder PC. (2005) Reprod Nutr Dev 45, 581-97. 